\title{
Características clínicas y factores asociados a ingreso a Unidad de Cuidado Intensivo de pacientes con trauma en un hospital de alta complejidad en Cali, Colombia
}

\author{
${ }^{1}$ Carlos A Ordoñez, ${ }^{2}$ Mónica A Morales García, ${ }^{3}$ Cecibel Cevallos, ${ }^{4}$ Jenny Marcela Vidal Carpio, ${ }^{5}$ Marisol Badiel
}

\begin{abstract}
RESUMEN
Introducción: Errase Colombia. la muerte por lesiones de causa externa es la segunda causa de muerte en Colombia y la primera en Cali. Parte de la atención de estos pacientes incluye la necesidad de procedimientos quirúrgicos y/o ingreso a Unidades de Cuidado Intensivo (UCI). Se describen las características de los pacientes que requirieron $\mathrm{UCl}$.
\end{abstract}

Material y métodos: A partir del Registro Internacional de Trauma de la Sociedad Panamericana de Trauma (ITR/SPTITSDP) en un hospital privado de primer nivel se revisan todos los registros consecutivos de los sujetos que ingresaron por lesión de causa externa con injury severity score (ISS) $\geq 9$ o con al menos 6 horas en observación-urgencias entre enero2012 a Diciembre-2014 y requirieron UCl.

Resultados: De 3791 ingresos, el $31.71 \%(n=1.202)$ correspondieron a sujetos que requirieron la $\mathrm{UCl}$. El $78.2 \%$ fueron hombres. La edad promedio fue $33.5 \pm 21$ años. El $43.1 \%$ ingresaron con ISS $\geq 15$. Solo el $16.4 \%$ de los lesionados tuvieron compromiso craneoencefálico, el $35.7 \%$ presentaron heridas penetrantes por arma de fuego o cortopunzantes. EL $69.88 \%$ $(n=840)$ fueron sometidos a cirugía. La mediana de estancia en UCI fue de 4 [IQR 2-7 días], el 65.56\% $(n=788)$ requirieron uso de ventilador. La mortalidad total fue de $11.2 \%$ (133/1202) vs $4.8 \%$ (123/1900) por fuera de UCI. EL RR para mortalidad fue del 2.3 de los sujetos que ingresan a $\mathrm{UCl}$ comparado con los que no. Al ingreso a urgencias, los factores predictores de necesidad de UCl fueron Glasgow coma scale (GCS) $<13(R R=5.77)$, ISS $>$ $15(R R=6.56)$, politraumatismo $(R R=1.86)$, politraumatismo sin trauma de cráneo $(R R=2.32)$, trauma craneoencefálico $(R R=2.55)$ y herida por arma de fuego $(R R=2.46)$.

Conclusión: De los sujetos con lesiones de causa externa, con trauma con compromiso moderado o severo que ingresaron a un hospital de primer nivel, el $31.71 \%$ requirieron una $\mathrm{UCl}$. La mortalidad fue del $11.2 \%$. Factores asociado al ingreso a $\mathrm{UCl}$

\footnotetext{
${ }^{1}$ Cirujano, ${ }^{2}$ Estadística, ${ }^{3,4}$ Cirujana de Trauma, ${ }^{5}$ Epidemiologa ${ }^{1}$ Intensivista, Fundación Valle del Lili. Director Departamento de Cirugía, Facultad de Salud, Universidad del Valle, Hospital Universitario del Valle, Cali, Colombia

${ }^{2,5}$ Departamento de Cirugía, Hospital Universitario del Valle Cali, Valle del Cauca, Colombia

${ }^{3,4}$ Departamento de Cirugía de Trauma y Emergencias Universidad del Valle, Cali, Valle del Cauca, Colombia

Corresponding Author:Carlos A Ordoñez, Cirujano, Intensivista Fundación Valle del Lili. Director Departamento de Cirugía Facultad de Salud, Universidad del Valle, Hospital Universitario del Valle, Cali, Colombia, Tel: +572319090, e-mail: ordonezcarlosa@ gmail.com
}

fueron GCS < 13, ISS > 15, politraumatismo, trauma craneoencefálico y herida por arma de fuego.

Palabras Clave: Indices de gravedad del trauma, Sistemas de datos, Trauma, Unidad de cuidados intensivos.

How to cite this article: Ordoñez CA, García MAM, Cevallos C Carpio JMV, Badiel M. Características clínicas y factores asociados a ingreso a Unidad de Cuidado Intensivo de pacientes con trauma en un hospital de alta complejidad en Cali, Colombia. Panam J Trauma Crit Care Emerg Surg 2018;7(1):1-3.

\section{Source of support: Nil}

Conflict of interest: None

\section{ABSTRACT}

Introduction: Violence continues to be one of the leading causes of mortality in Colombia. We sought to describe and analyze a series of trauma patients that required intensive unit care in a high-complexity center in Cali, Colombia.

Materials and methods: Using data from the Panamerican Trauma Registry, we reviewed all victims of trauma that were admitted with an injury severity score higher or equal to 9 or with at least 6 hours of observation in the emergency room and required intensive care unit admission from January 2002 to December 2014

Results: A total of 3,791 patients were included in the registry during the period observed, of which 1,202 corresponded to patients that required intensive care unit admission; $78.2 \%$ were male. Mean (standard deviation) age was 33.5 (21) years. A total of $43.1 \%$ suffered trauma with an injury severity score higher or equal to $15 ; 16.4 \%$ suffered traumatic brain injury and $35.7 \%$ suffered penetrating injuries. A total of 840 patients were taken to emergency surgery and 788 required mechanical ventilation. Median (interquartile range) of intensive care unit stay was $4(2-7)$ days. Mortality in the intensive care unit was 11.2 vs 4.8 outside the intensive care unit. Multivariate regression analysis showed that factors associated with intensive care unit admission were a Glasgow coma scale $<13$, an injury severity score $>15$, the presence of polytrauma or polytrauma without traumatic brain injury, traumatic brain injury alone, and the presence of gunshot wounds.

Conclusion: Our multivariate analysis showed several factors associated with intensive care unit admission in a large trauma population.

Keywords: Injury severity indices of trauma, Intensive care for trauma, Trauma data systems.

\section{INTRODUCCIÓN}

El paciente traumatizado actualmente plantea un problema importante en el mundo, tanto de salud pública 
como económica y social, ya que su manejo supone un reto importante para los servicios de salud derivado del alto costo en su atención y el impacto social que tiene la afectación de estos pacientes. De ello deriva la importancia de crear instrumentos que permitan homogenizar su evaluación, manejo, pronóstico y calidad de vida que le espera lo más cercano a la realidad, para preparar el equipo médico de emergencias y realizar un diagnóstico y tratamiento oportuno. ${ }^{1-4}$

La muerte por lesiones de causa externa es la segunda causa de muerte en Colombia y la primera en Cali. ${ }^{5}$ En Colombia se han adaptado estrategias de manejo desde la admisión de los pacientes en que se realizan maniobras de reanimación tempranamente y han cambiado los indicadores de mortalidad en pacientes moderada y severamente comprometidos. ${ }^{6,7}$ El manejo en si mismo de la emergencia, ${ }^{8}$ la ganancia en habilidades del manejo quirúrgico del trauma, y también en el manejo del paciente politraumatizado en unidades de cuidado intensivo contribuyen a dichos resultados, de tal manera que incluso desde hace más de cuatro décadas se propuso el modelo de unidades de cuidado intensivo para trauma ${ }^{9}$ y hoy son una realidad en muchos sistemas de trauma. ${ }^{6} \mathrm{La}$ tendencia en el mundo es que la atención de estos pacientes incluye la necesidad de procedimientos quirúrgicos y/o ingreso a Unidades de Cuidado Intensivo (UCI) ha cambiado a través de los años optimizando los recursos, más pacientes requieren menos días de ventilación o estancia, menores complicaicones, realizando una mejor evaluación de los pacientes con ayudas diagnósticas, mejorando las condiciones desde la sala de emergencias a cirugía con la reanimación. ${ }^{10,11}$ Por ello, es pertinente describir las características de los pacientes politraumatizados que requirieron UCI que poco se ha descrito en Colombia para lograr identificar desde el ingreso a urgencias, cuáles son los que la requerirán.

\section{MATERIAL Y MÉTODOS}

A partir del Registro Internacional de Trauma de la Sociedad Panamericana de Trauma (ITR/SPT-ITSDP) en un hospital privado de tercer nivel se revisan todos los registros consecutivos de los sujetos que ingresaron por lesión de causa externa entre enero-2012 a Diciembre-2014.

Se definió como paciente con compromiso moderado a severo si al ingreso se presentaba con ISS $\geq 9$ o con al menos 6 horas en observación-urgencias y requirieron UCI. Se excluyeron registros con datos incompletos de la hospitalización.

Análisis: Se realiza un análisis descriptivo, según el comportamiento de las variables continuas en cuanto a la normalidad de su distribución se estima promedios y desviación estándar y en caso contrario, mediana con rangos intercuartílicos. Las variables dicotómicas y categóricas se expresan en proporciones. Se realiza un análisis multivariado para identificar los posibles factores asociados a la necesidad de requerir UCI. Los cálculos fueron realizados en $R$.

El estudio fue aprobado por el comité de ética de la investigación.

\section{RESULTADOS}

De 3791 ingresos, el $31.71 \%(n=1.202)$ correspondieron a sujetos que requirieron la UCI. El 78.2\% fueron hombres. La edad promedio fue $33.5 \pm 21$ años. El 43.1\% ingresaron con ISS $\geq 15$. Solo el $16.4 \%$ de los lesionados tuvieron compromiso craneoencefálico, el $35.7 \%$ presentaron heridas penetrantes por arma de fuego o cortopunzantes. El 69.88\% (n=840) fueron sometidos a cirugía. La mediana de estancia en UCI fue de 4 [IQR 2-7 días], y la estancia hospitalaria total fue de 7 [IQR 4-15 días], el 65.56\% $(\mathrm{n}=788)$ requirieron uso de ventilador. La mortalidad total fuede $11.2 \%(133 / 1202)$ vs $4.8 \%(123 / 1900)$ por fuera de UCI estadísticamente significativa $(\mathrm{p}<0.0001)$. Ver Tabla 1. EL RR para mortalidad fue del 2.3 de los sujetos que ingresan a UCI comparado con los que no. Al ingreso a urgencias, los factores predictores de necesidad de UCI fueron GCS $<13(\mathrm{RR}=5.77), \mathrm{ISS}>15(\mathrm{RR}=6.56)$, politraumatismo $(\mathrm{RR}=1.86)$, politraumatismo sin trauma de cráneo $(R R=2.32)$, trauma craneoencefálico $(R R=2.55)$ y herida por arma de fuego $(R R=2.46)$. Ver Tabla 2 .

Tabla 1: Características generales

\begin{tabular}{ll}
\hline & $N=1,202$ \\
\hline Edad, años & $33.5 \pm 21$ años \\
Hombres, \% & 78.2 \\
ISS $\geq 15, \%$ & 43.1 \\
ISS $>9-14, \%$ & 32.7 \\
Necesidad de Cirugía, \% & 69.88 \\
Estancia, mediana [IQR] & \\
$\quad$ UCI & 4 [IQR 2-7 días] \\
$\quad$ Hospitalaria total & 7 [IQR 4-15 días] \\
Necesidad de ventilador en UCI, \% & 65.56 \\
Tasa de mortalidad, \% & \\
$\quad$ UCI & 11.2 \\
Sin UCl & 4.8 \\
\hline
\end{tabular}

Tabla 2: Riesgo relativo para los sujetos que ingresaron a $\mathrm{UCI}$ comparados con quienes no

\begin{tabular}{ll}
\hline & $R R[I C 95 \%]$ \\
\hline GCS $<13$ & $5.77[4.90-6.79]$ \\
ISS $>15$ & $6.56[5.60-7.70]$ \\
Politraumatismo sin Trauma craneano & $2.32[2.05-2.64]$ \\
Politraumatismo con Trauma & $1.86[1.73-2.01]$ \\
craneoencefálico & \\
Trauma solo de cráneo & $2.55[2.11-3.10]$ \\
Herida por arma de fuego & $2.46[2.16-2.81]$ \\
\hline
\end{tabular}




\section{CONCLUSIÓN}

De los sujetos con lesiones de causa externa, con trauma con compromiso moderado o severo que ingresaron a un hospital de primer nivel, el $31.71 \%$ requirieron una UCI. En otras series se describe que el uso de UCI es común y que va entre el 25 al 30\% de los sujetos traumatizado requieren de UCI. ${ }^{8}$ Ello indica que en la experiencia que se presenta está acorde con lo que se presenta en la literatura. La mortalidad fue del $11.2 \%$, una tasa por debajo de otras series que estiman que hasta cerca de un $17 \%$ de mortalidad en sujetos que ingresaron a UCI y se complicaron. ${ }^{8}$ Factores asociado al ingreso a UCI fueron GCS $<13$, ISS $>15$, politraumatismo, trauma craneoencefálico y herida por arma de fuego.

\section{DISCUSIÓN}

El traumatismo es quizás la enfermedad más difícil de cuantificar debido a la gran variabilidad de asociaciones de lesiones graves que comprometen la vida o causan incapacidad permanente. ${ }^{12}$

Un estudio realizado por el grupo de Chico $\mathrm{M}$, sobre las características de pacientes traumatizados ingresados en UCI, en España, reportó que la media en años fue de 47,1 $\pm 19,02$, mientras que la edad promedio que se obtuvo en este trabajo fue $33.5 \pm 21$ años, estas diferencias se pueden atribuir a las diferentes etiologías del trauma en estos países. En cuanto al sexo, no presento diferencia en estos dos trabajos, el 79\% del sexo masculino en España y el 78.2\% Cali. ${ }^{13}$

El Injury Severity Score reportado fue de 22,2 $\pm 12,1$, mientras que en el presente trabajo el $43.1 \%$ de los pacientes ingresaron con ISS $\geq 15$, lo que traduce que en ambos trabajos los pacientes ingresados tuvieron un trauma grave. ${ }^{13}$

El presente trabajo evidenció que sólo el 16.4\% de los pacientes traumatizados tuvieron compromiso craneoencefálico, y el $35.7 \%$ presentaron heridas penetrantes por arma de fuego o cortopunzantes; datos totalmente opuestos con los resultados de Chico $\mathrm{M}$ y Peinado J, quiénes reportan que las tasas de mortalidad más elevadas en las Unidades de Cuidados Intensivos (UCI), en los pacientes con enfermedad traumática severa y grave, se produce inicialmente después del ingreso, debido a los traumatismos craneoencefálicos cerrados, fallo respiratorio agudo o shock hemorrágico, pudiendo deberse principalmente a que las causas más frecuentes de trauma en estos trabajos fueron accidentes de tránsito, caídas y precipitacione. ${ }^{13-14}$

Las estancia en UCI reportada por Chico M, fue una mediana $5^{3-13}$ días vs la obtenida en este trabajo que fue de 4 [IQR 2-7 días], es decir una diferencia de un día, que matemáticamente hablando representa más costos, pudiendo deberse en parte a que la edad promedio del grupo de estudio de Chico es mayor con 10 años, con la posibilidad de que éstos pacientes tengan más comorbilidades asociadas. ${ }^{13}$

Todos los estudios y la elaboración de registros en Medicina Intensiva contribuyen a mejorar la asistencia sanitaria de los pacientes traumatizados que precisan ingreso en la UCI, a través principalmente de un conocimiento de las características y las maniobras terapéuticas que se realizan según cada patología.

\section{REFERENCIAS}

1. Flismina R. Incidencia de politraumatismo en pacientes adultos, servicio de emergencia. Hospital Central Universitario Dr. Antonio María Pineda Barquisimeto. Julio 2001Julio 2002. 2003. [último acceso julio 2016]. Disponible en: http://bibmed.ucla.edu.ve/edocs_bmucla/TextoCompleto/ TW0700D422003.pdf

2. Van Natta T, Morris J. Calificación de lesiones y resultados de los traumas. In: Mattox K, Feliciano D, Moore E. Trauma. 4ed. México, DF: McGraw-Hill Interamericana; 2001. pp. 73- 86.

3. Dellinger E. Use of scoring systems to assess patients with surgical sepsis. Surg Clin North Am 1988;68:123-145.

4. Champion HR, Sacco WJ, Gainer PS, Patow SM. The effect of medical direction on trauma triage. J Trauma 1988 Feb;28(2): 235-239.

5. Departamento Administrativo de Planeación Municipal. Plan de Desarrollo Municipal 2012D2015 de Santiago-CaliDA: una ciudad para todos (on line). Santiago de Cali, Colombia; 2012 http://www.cali.gov.co/publicaciones.php?id=44418 2012 (cited 2016).

6. Ordoñez CA, Badiel M, Sánchez ÁI, Granados M, García AF, Ospina G, Blanco G, Parra V, Gutiérrez-Martínez MI, Peitzman $\mathrm{AB}$, et al. Improving mortality predictions in trauma patients undergoing damage control strategies. The American Surgeon 2011 Jun;77(6):778-782.

7. Ordoñez CA, Parra M, Badiel M, Pino LL, Calle AA, Miñan FD, Mejia DA, Rojas-Mirquez JC, Bonilla-Escobar FJ, Puyana JC. Damage control resuscitation and surgery: associated factors of mortality in penetrating trauma. J Am Coll Surg 2015; 221(4):e140-e141.

8. Magnone S, Allegri A, Belotti E, Castelli CC, Ceresoli M, Coccolini F, Manfredi R, Merli C, Palamara F, Piazzalunga D, et al. Impact of ATLS guidelines, trauma team introduction, and 24-hour mortality due to severe trauma in a busy, metropolitan Italian hospital: A case control study. Ulus Travma Acil Cerrahi Derg 2016 May;22(3):242-246.

9. Lowe RJ, Baker RJ. Organization and function of trauma care units. J Trauma 1973 Apr; 13(4):285-290.

10. Jansen JO, Lendrum RA, Morrison JJ. Trauma care in Scotland: The role of major trauma centres, trauma units, and local emergency hospitals. The Surgeon 2016;14(5):241-244.

11. Böhmer AB, Poels $M$, Kleinbrahm $K$, Lefering R, Paffrath $T$, Bouillon B, Defosse JM, Gerbershagen MU, Wappler F, et al. Change of initial and ICU treatment over time in trauma patients. An analysis from the TraumaRegister DGU ${ }^{\circledR}$. Langenbecks Arch Surg 2016 Jun;401(4):531-540.

12. Prin M, Li G. Complications and in-hospital mortality in trauma patients treated in intensive care units in the United States, 2013. Inj Epidemiol 2016 Dec;3(1):18.

13. Chico-Fernández M, Llompart-Pou JA, Guerrero-López F, Sánchez-Casado M, García-Sáez I, Mayor-García MD, Egea-Guerrero J, Fernández-Ortega JF, Bueno-González A, González-Robledo J, et al. Epidemiology of severe trauma in Spain. Registry of trauma in the ICU (RETRAUCI). Pilot phase. Med Intensiva 2016 Aug-Sep;40(6):327-347.

14. Peinado J. Tratamiento del paciente traumatizado en UCI, Hospital de Poniente, 2012. 